\title{
Photonic spin Hall effect in hyperbolic metamaterials at visible wavelengths
}

\author{
Takayama, Osamu; Sukham, Johneph; Malureanu, Radu; Lavrinenko, Andrei V.; Puentes, Graciana
}

Published in:

Optics Letters

Link to article, DOI:

10.1364/OL.43.004602

Publication date:

2018

Document Version

Peer reviewed version

Link back to DTU Orbit

Citation (APA):

Takayama, O., Sukham, J., Malureanu, R., Lavrinenko, A. V., \& Puentes, G. (2018). Photonic spin Hall effect in hyperbolic metamaterials at visible wavelengths. Optics Letters, 43(19), 4602-5.

https://doi.org/10.1364/OL.43.004602

\section{General rights}

Copyright and moral rights for the publications made accessible in the public portal are retained by the authors and/or other copyright owners and it is a condition of accessing publications that users recognise and abide by the legal requirements associated with these rights.

- Users may download and print one copy of any publication from the public portal for the purpose of private study or research.

- You may not further distribute the material or use it for any profit-making activity or commercial gain

- You may freely distribute the URL identifying the publication in the public portal

If you believe that this document breaches copyright please contact us providing details, and we will remove access to the work immediately and investigate your claim 


\title{
Photonic spin Hall effect in hyperbolic metamaterials at visible
}

\author{
Osamu Takayama ${ }^{1, *}$, Johneph SuKham ${ }^{1}$, Radu Malureanu ${ }^{1}$, Andrei V. \\ LaVRINENKo $^{1}$, And Graciana Puentes ${ }^{2,3}$ \\ ${ }^{1}$ DTU Fotonik - Department of Photonics Engineering, Technical University of Denmark, Ørsteds Plads 343, DK-2800 Kgs. Lyngby, Denmark \\ ${ }^{2}$ Universidad de Buenos Aires. Facultad de Ciencias Exactas y Naturales. Departamento de Física. Buenos Aires, Argentina \\ ${ }^{3}$ CONICET-Universidad de Buenos Aires. Instituto de Física de Buenos Aires (IFIBA). Buenos Aires, Argentina \\ *Corresponding author: otak@fotonik.dtu.dk, gpuentes@df.uba.ar
}

Compiled July 11, 2018

Photonic spin Hall effect in transmission is a transverse beam shift of the out-coming beam depending on polarization of the in-coming beam. The effect can be significantly enhanced by materials with high anisotropy. We report the first experimental demonstration of the photonic spin Hall effect in a multilayer hyperbolic metamaterial at visible wavelengths (wavelengths of $520 \mathrm{~nm}$ and $633 \mathrm{~nm}$ ). The metamaterial is composed of alternating layers of gold and alumina with deeplysubwavelength thicknesses, exhibiting extremely large anisotropy. The angle resolved polarimetric measurements showed the shift of $\mathbf{1 6 5} \mu \mathrm{m}$ for the metamaterial of $176 \mathrm{~nm}$ in thickness. Additionally the transverse beam shift is extremely sensitive to the variations of the incident angle changing theoretically by $270 \mu \mathrm{m}$ with one milli-radian $\left(0.057^{\circ}\right)$. These features can lead to minituarized spin Hall switches and filters with high angular resolution. (C) 2018 Optical Society of America under the terms of the OSA Open Access Publishing Agreement

OCIS codes: (160.3918) Metamaterials; (240.5440) Polarizationselective devices; (260.0260) Physical optics.

http://dx.doi.org/10.1364/optica.XX.XXXXXX

The photonic spin Hall effect [1] or spin Hall effect of light [2] is the photonic analogue of the spin Hall effect occurring with charge carriers in solid-state systems. Typically this phenomenon takes place when a light beam refracts at an air-glass interface, or when it is projected onto an oblique plane, the latter effect being known as the geometric spin Hall effect of light. In general the photonic spin Hall effect leads to a polarizationdependent transverse $y$-shift of a light peak intensity [3-6]. An example of the latter effect is the transverse Imbert-Federov beam shift [3], which happens for paraxial beams reflected or refracted at a sharp inhomogeneity of an isotropic optical interface [4-7]. Potential applications of the photonic spin Hall effect in spin-dependent beam splitters, optical diodes [8] and surface sensors $[9,10]$ are considered in various fields in photonics such as nanophotonics, plasmonics, metamaterials, topological optics and quantum optics $[9,11]$.

Photonic spin Hall effect has been studied in reflection and transmission with various materials and geometrical settings. In the reflection configuration, the effect has been studied experimentally on interfaces of uniaxial dielectrics $\left(\mathrm{LiNbO}_{3}\right)[11], \mathrm{BK}-7$ glasses [12], glass prisms [13], metal (Ag) films [14], magnetic films [15], dielectric multilayers [16] and topological insulators [17]. In the case of the transmission configuration, the photonic spin Hall effect has been experimentally demonstrated in quarts crystals [18], anisotropic polymers [19], metal (Au) films [20], liquid crystals [21] and dielectric spheres [22].

Recently, metasurfaces and metamaterials, artificially engineered subwavelength surface and volume structures, have stimulated significant interest thanks to their flexible design parameters [23]. A subclass of metamaterials - hyperbolic metamaterials (HMM) exhibit extreme anisotropy with hyperbolic dispersion profiles in the wavevector space. Conventionally, HMMs take the form of a multilayer of alternating metal and dielectric thin films [24-26], trenches [27-29] or metallic nanowires [30, 31]. In the typical cases HMMs possess uniaxial anistropy, and their optical properties are characterized by effective ordinary and extraordinary permittivities, $\varepsilon_{0}$ and $\varepsilon_{e}$, respectively, as depicted in Fig 1. Depending on the sign of $\varepsilon_{0}$ and $\varepsilon_{e}$, a metamaterial can be categorized as so-called type I ( $\varepsilon_{0}>0$ and $\left.\varepsilon_{e}<0\right)$ or type II $\operatorname{HMM}\left(\varepsilon_{o}<0\right.$ and $\left.\varepsilon_{e}>0\right)[32,33]$. Such unique optical properties of HMMs have led to various applications, sub-diffraction imaging [34] and sensing [35-37], to name a few.

The spin Hall effect in HMMs has been theoretically studied recently $[38,39]$. Experimental observation of the spin Hall effect in HMMs has been reported so far only for microwave frequencies [40]. The hyperbolic dispersion obtained with electronic components, such as capacitors and inductors, helps to steer directional surface waves by input polarization states. 


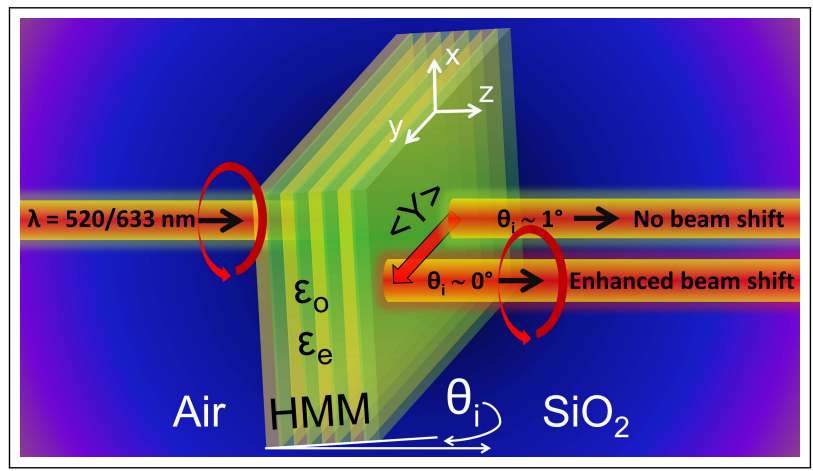

Fig. 1. Schematic illustration of the spin Hall effect. The transverse beam shift along the y-axis denoted as $\langle Y\rangle$ is switched by incident angle $\theta_{i}$. The unit cell of the HMM structure consists of $\mathrm{Al}_{2} \mathrm{O}_{3}(10 \mathrm{~nm})-\mathrm{APTMS}(1 \mathrm{~nm})-\mathrm{Au}(10 \mathrm{~nm})$ APTMS $(1 \mathrm{~nm})$. The HMM contains eight periods with the total thickness of $176 \mathrm{~nm}$.

Here, we experimentally demonstrate extreme angle sensitivity and enhancement of the spin-Hall effect of light in HMMs in the visible regime. The effect is shown in the transmission configuration with a few hundreds nanometers thick HMM composed of alternating layers of metal and dielectric as illustrated in Fig. 1. The transverse beam shift in our HMM setting is very sensitive to the angle of incidence, drastically changing from almost no beam shift to a few hundred microns by the change of $\approx 0.003 \mathrm{rad}\left(\approx 0.17^{\circ}\right)$ in the incident angle. The large photonic spin Hall enhancement in a such thin structure and extreme angular sensitivity can be exploited in compact spin Hall devices which enable manipulation of photons by polarization.

The HMM sample with the total thickness of $176 \mathrm{~nm}$ contains eight gold-alumina periods deposited on a $500 \mu \mathrm{m}$ thick glass substrate. More detailed, one period of the HMM structure consists of four layers: $\mathrm{Al}_{2} \mathrm{O}_{3}(10 \mathrm{~nm})-\mathrm{APTMS}(1 \mathrm{~nm})-\mathrm{Au}(10$ $\mathrm{nm})-\mathrm{APTMS}(1 \mathrm{~nm})$. APTMS, which stands for Amino Propyl Tri Methoxy Silane, is an almost loss-free adhesion layer advantageous for highly localized propagating plasmon modes [26]. The $\mathrm{Au}$ layer was sputtered, and $\mathrm{Al}_{2} \mathrm{O}_{3}$ layer was deposited by atomic layer deposition. The treatment of HMMs as homogenized uniaxial media with effective permittivities are based on the effective media approximation [41], which is assumed to be valid under the condition that the thicknesses of individual layers are deeply subwavelength. The unit cell is $\Lambda=22 \mathrm{~nm}$, and normalized by the wavelength interval of $\lambda=500-700 \mathrm{~nm}$, provides the ratio of $\Lambda / \lambda=1 / 22.7-1 / 31.8$. Thus application of the effective media approximation is our case is justified.

The ordinary and extraordinary permitivities of the HMM, denoted as $\varepsilon_{o}$ and $\varepsilon_{e}$ respectively, are calculated as [28]

$$
\begin{gathered}
\varepsilon_{o}=f_{A u} \cdot \varepsilon_{A u}+f_{A l_{2} O_{3}} \cdot \varepsilon_{A l_{2} O_{3}}+f_{A P T M S} \cdot \varepsilon_{d_{A P T M S}} \\
\varepsilon_{e}=\left(\frac{f_{A u}}{\varepsilon_{A u}}+\frac{f_{A l_{2} O_{3}}}{\varepsilon_{A l_{2} O_{3}}}+\frac{f_{A P T M S}}{\varepsilon_{A P T M S}}\right)^{-1},
\end{gathered}
$$

where $\varepsilon_{m}$ and $\varepsilon_{d}$ are the permittivities of metal and dielectric, and $f_{m}$ and $f_{d}$ are the volume fractions of metal and dielectric, respectively. The permittivity of the Au film, $\varepsilon_{m}$, is characterized by the Drude-Lorentz model with the thickness-dependent correction [29]. The refractive index of APTMS is 1.46 [30]. Fig. 2 shows dispersion of the HMM effective permittivities in the visible range. Our HMM structure has a zero crossing wavelength for $\varepsilon_{o}$ around $\lambda=500 \mathrm{~nm}$ and further to the red wavelengths becomes type II HMMs $\left(\varepsilon_{o}<0\right.$ and $\left.\varepsilon_{e}>0\right)$.

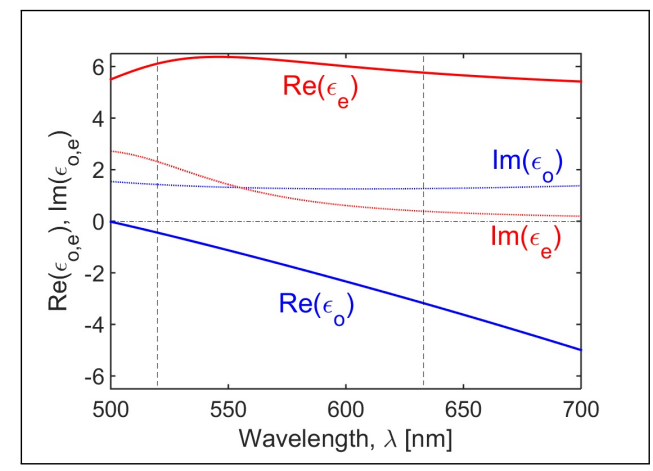

Fig. 2. Effective permittivities of multilayer HMM structure composed of a unit cell of $\mathrm{Al}_{2} \mathrm{O}_{3}(10 \mathrm{~nm})$-APTMS(1 nm)-Au(10 $\mathrm{nm})$-APTMS(1 nm) calculated by the effective media approximation. In the visible wavelength interval, the structure behaves as type II $\left(\varepsilon_{o}<0\right.$ and $\left.\varepsilon_{e}>0\right) \mathrm{HMM}$. The wavelengths $\lambda$ $=520 \mathrm{~nm}$ and $633 \mathrm{~nm}$ at which we conducted experiments are noted by the vertical dashed lines.

In order to understand the behavior of the spin Hall beam shift in HMMs, we conducted simulation based on a theory developed by T. Tang et al. [41,42] with realistic parameters of fabricated HMMs presented in Fig. 2. The whole $\mathrm{HMM}-\mathrm{SiO}_{2}$ substrate structure has air as the ambient medium as shown in Fig. 1. We assume the incident light is impinging on the HMM structure in the $\mathrm{y}-\mathrm{z}$ plane with incident angle $\theta_{i}$. The relative permittivities of the media in regions 1-5 are denoted by $\epsilon_{i}(i=1,2,3,4,5)$ respectively, where $\epsilon_{1}=\epsilon_{2}=\epsilon_{5}=1$ (air), $\epsilon_{4}$ corresponds to the $\mathrm{SiO}_{2}$ substrate and $\epsilon_{3}$ corresponds to the HMM. The HMM is assumed to be non-magnetic and uniaxial anisotropic with a relative permittivity tensor $\left(\epsilon_{3}\right)$ :

$$
\epsilon_{3}=\left(\begin{array}{ccc}
\epsilon_{o} & 0 & 0 \\
0 & \epsilon_{o} & 0 \\
0 & 0 & \epsilon_{e}
\end{array}\right)
$$

Considering the input Gaussian beam of waist $\omega_{0}$ :

$$
E_{H, V}(x, y)=\frac{\omega_{0}}{\sqrt{2 \pi}} \exp -\frac{\omega_{0}^{2}\left(k_{x}^{2}+k_{y}^{2}\right)}{4}
$$

we can define the transverse beam shifts after transmission through the structure in the form:

$$
\eta_{H, V}^{ \pm}=\frac{x\left|E_{H, V}\right|^{ \pm}}{\left|E_{H, V}\right|^{ \pm}}
$$

where $\eta^{ \pm}$indicate transverse shifts for the right hand circular (RHC) and left hand circular (LHC) polarizations. The transverse shifts include $z$-dependent and $z$-independent terms which represent spatial and angular transverse shifts, respectively [31]. Here, we focus on the spatial transverse shift of transmitted light through the HMM waveguide, which takes the form [32]:

$$
<Y>= \pm \frac{k_{1} \omega_{0}^{2}\left(t_{s}^{2} \frac{\cos \left(\theta_{t}\right)}{\sin \left(\theta_{i}\right)}-t_{s} t_{p} \cot \left(\theta_{i}\right)\right)}{k_{1}^{2} \omega_{0}^{2} t_{s}^{2}+\cos ^{2}\left(\theta_{i}\right)\left(t_{s} \frac{\cos \left(\theta_{t}\right)}{\cos \left(\theta_{i}\right)}-t_{p}\right)^{2}+\left(\frac{d t_{s}}{d \theta_{i}}\right)^{2}}
$$


where $k_{1}=n_{1} k=n_{1} \frac{2 \pi}{\lambda}$ with $n_{1}=1$ (air), $t_{s, p}$ are the transmission amplitudes for the $s, p$ modes, respectively [41], $\theta_{t}$ is the transmission angle. We consider $\left(\frac{d t_{s}}{d \theta_{i}}\right) \approx 0$ for a large beam waist and $\theta_{t}=0$, assuming transmission along the laser beam axis.

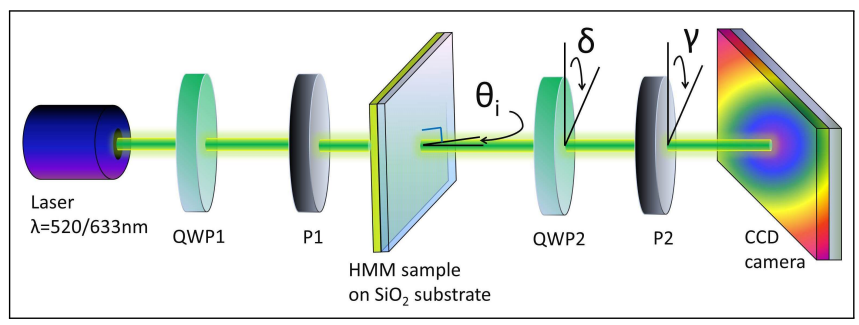

Fig. 3. Schematic illustration of polarimetric measurement setup. P1 and P2 represent double Glan-Laser polarizers (Thorlabs GL10), QWP1 and QWP2 are quarter wave plates. Laser is either a He-Ne laser (Melles Griot 05-LHR-111) or diode laser (Thorlabs LP 520-MF-100) with wavelength of $633 \mathrm{~nm}$ and 520 $\mathrm{nm}$, respectively. The CCD camera is Thorlabs WFS150-5C.

To demonstrate the angular sensitivity of the photonic spin Hall shift in the HMM, we performed a series of characterizations using the polarimetric setup shown in Fig. 3. The transverse beam shift can be measured by polarimetric and quantum weak measurement $[20,21,43]$. As a source of an incident Gaussian beam, we employed a He-Ne laser with wavelength $\lambda=633$ $\mathrm{nm}$, and a diode laser with wavelength $\lambda=520 \mathrm{~nm}$. The laser radiation was collimated using a microscope objective lens. We measured the anisotropic phase difference $\Phi_{0}$ versus angle $\theta_{i}$ with the Stokes polarimetry method [20]. The input polarization state (RHC) is prepared using a Quarter Wave-Plate (QWP) followed by a Glan-Thompson polarized (P1). The phase difference can be obtained via the Stokes parameters using the expression:

$$
\Phi_{0}=\arctan \left(S_{3} / S_{2}\right),
$$

where $S_{3}=I\left(90^{\circ}, 45^{\circ}\right)-I\left(90^{\circ}, 135^{\circ}\right)$ is the normalized Stokes parameter for the circular polarization, and $S_{2}=I\left(0^{\circ}, 45^{\circ}\right)-$ $I\left(0^{\circ}, 135^{\circ}\right)$ is the normalized Stokes parameter in the diagonal basis, where normalization factor $S_{0}$ is given by the total intensity of the beam. $\delta$ and $\alpha$ of $I(\delta, \alpha)$ correspond to the retardation angle of quarter wave plate QWP2, and the rotation angle of polarizer P2, respectively. The measured phase using Eq.7 is wrapped in the range $(-\pi, \pi)$. In order to determine the unwrapped phase difference we use the unwrapping algorithm [20], with a tolerance set to $0.01 \mathrm{rad}$.

The transverse beam shift, $\langle Y\rangle$, is found from the measured phase, $\Phi_{0}$,

$$
<>=\frac{1}{k} \cot \left(\pi / 2-\theta_{i}\right)\left[-\sigma\left(1-\cos \Phi_{0}\right)+\chi \sin \Phi_{0}\right],
$$

where $\sigma$ and $\chi$ are the Stokes parameters in the circular and diagonal basis, respectively. They are given by $\sigma=2 \operatorname{Im}\left(\alpha^{*} \beta\right)$, and $\chi=2 \operatorname{Re}\left(\alpha^{*} \beta\right)$ from the Jones vector $|\psi\rangle=\left(\begin{array}{l}\alpha \\ \beta\end{array}\right)$ of the incident beam, respectively. The incident beam has the RHC polarization, that is, $\alpha=\frac{1}{\sqrt{2}}, \beta=\frac{i}{\sqrt{2}}$.

Fig. 4 shows the experimental points of the beam shifts for wavelengths $\lambda=520 \mathrm{~nm}$ and $\lambda=633 \mathrm{~nm}$ versus the simulated results, where losses of the HMM were taken into account. The effective permittivitties are $\varepsilon_{0}=-2.9460+1.2163 \mathrm{i}$ and $\varepsilon_{e}=5.5063+$ $0.3399 \mathrm{i}$ for $\lambda=633 \mathrm{~nm}$, and $\varepsilon_{o}=-0.3257+1.3664 \mathrm{i}$ and $\varepsilon_{e}=5.8847$

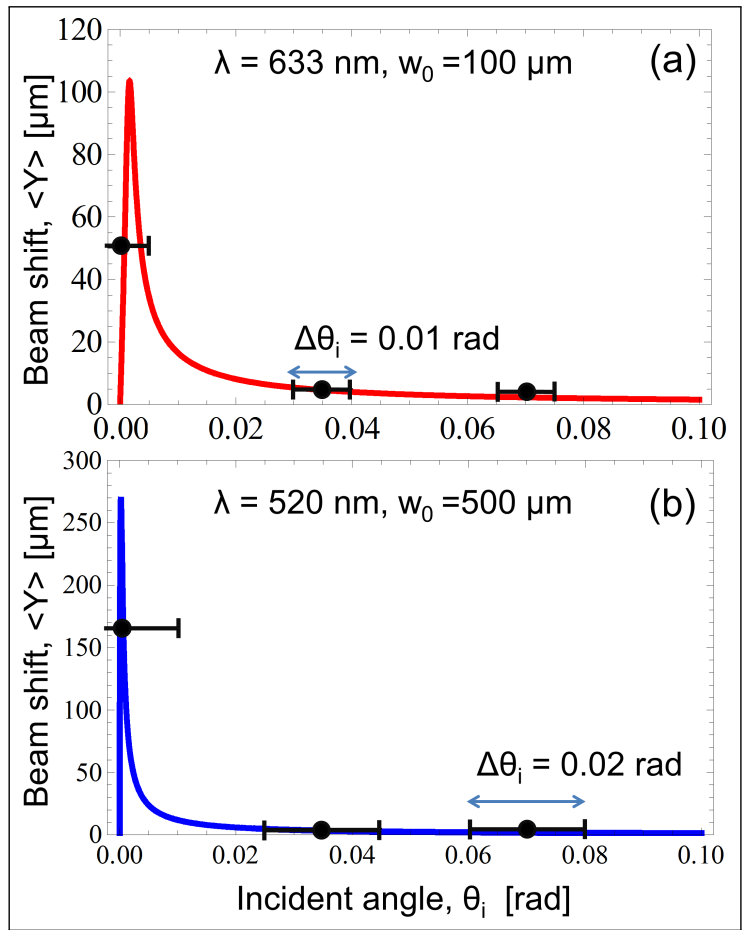

Fig. 4. Measured and simulated (solid lines) spin Hall transverse shift, $\langle Y>$, for (a) $\lambda=633 \mathrm{~nm}$ and (b) $\lambda=520 \mathrm{~nm}$, respectively. Simulation results in (a) and (b) are fitted in terms of beam width $w_{0}$. Note that there is an estimated beam divergence of $\Delta \theta_{i}=0.01 \mathrm{rad}$ for $\lambda=633 \mathrm{~nm}$ and $\Delta \theta_{i}=0.02 \mathrm{rad}$ for $\lambda$ $=520 \mathrm{~nm}$ indicated as lateral error bars, respectively.

$+1.9963 \mathrm{i}$ for $\lambda=520 \mathrm{~nm}$, respectively, see Fig. 2. Here we can observe the peculiarities of the photonic spin Hall effect in HMM structures in the transmission configuration. The tranverse beam shift in HMMs is extremely sensitive to the incident angle: its variation from $\theta_{i}=0 \mathrm{rad}$ to merely $\theta_{i}=0.003 \mathrm{rad}\left(0.17^{\circ}\right)$ for $\lambda=$ $633 \mathrm{~nm}$ [Fig. 4(a)] induces the giant beam shift of a few hundreds microns, exhibiting sensitivity in the milli-radian range. The angular variation from $\theta_{i}=0.003 \mathrm{rad}$ to above also significantly changes the beam shift from $\langle Y\rangle=105 \mu \mathrm{m}$ to merely $\langle Y\rangle$ $=10 \mu \mathrm{m}$, what is almost one order of magnitude difference [Fig. 4(a)]. The sharp peak of the beam shift is attributed to large anisotropy of HMMs, $\varepsilon_{e}-\varepsilon_{0}$, as we also saw such behavior in dielectric media [21]. In the case of $\lambda=520 \mathrm{~nm}$ with a larger beam diameter [Fig. 4(b)], the beams shift exhibits even sharper resonance and, consequently, enhanced angular sensitivity: the peak shift of $\langle Y\rangle=270 \mu \mathrm{m}$ is achieved by the incident angle change of $0.001 \operatorname{rad}\left(0.057^{\circ}\right)$ only $\left(\approx 4700 \mu \mathrm{m} /{ }^{\circ}\right)$. When the incident angle becomes larger, for instance, $\theta_{i}=0.01 \mathrm{rad}\left(0.57^{\circ}\right)$, the beam shift drastically drops to $\langle Y\rangle=10 \mu \mathrm{m}$ and less.

The experimental results are in the full quantitative agreement with the simulated values taking into account beams divergence and setups errors. The beam divergence of the $\mathrm{He}-\mathrm{Ne}$ laser is estimated to be about $\Delta \theta_{i}=0.01 \mathrm{rad}\left(0.57^{\circ}\right)$ and of the green diode laser is about $\Delta \theta_{i}=0.02 \mathrm{rad}\left(1.14^{\circ}\right)$. Additionally, the angular resolution is limited by the rotating mount with error about $0.0017 \mathrm{rad}\left(0.1^{\circ}\right)$. For $\lambda=633 \mathrm{~nm}$ [Fig. 4(a)], the experimental transverse beam shift under the normal incidence reaches $\langle Y\rangle=50 \mu \mathrm{m}$. However, when the incident angle is tilted by merely $\theta_{i}=0.035 \mathrm{rad}\left(\approx 2^{\circ}\right)$, the transverse beam shift dramatically drops down to $\langle Y\rangle=5 \mu \mathrm{m}$. Such results confirm 
that the transverse beam shift can be tuned within one order of magnitude range by small angular variations demonstrating extreme angular sensitivity of the spin Hall effect in HMMs. The waist used for the simulations $\left(w_{0}=100 \mu \mathrm{m}\right)$ is measured experimentally.

In the case of $\lambda=520 \mathrm{~nm}$, we observe $\langle Y\rangle=165 \mu \mathrm{m}$ for $\theta_{i}=0 \mathrm{rad}$ as shown in Fig. 4(b). At $\theta_{i}=0.035 \mathrm{rad}(\approx 2$ $\left.{ }^{\circ}\right)$, the beam shift decreases to $\langle Y\rangle=5 \mu \mathrm{m}$. This extremely high angular sensitivity makes a striking contrast to previously observed spin Hall effect in dielectric anisotropic media, such as a quartz crystal $\left(\approx 150 \mu \mathrm{m} / 5^{\circ}=30 \mu \mathrm{m} /{ }^{\circ}\right)$ [20] and polymer film $\left(\approx 250 \mu \mathrm{m} / 20^{\circ}=12.5 \mu \mathrm{m} /{ }^{\circ}\right)$ [21], where the beam shift of the same magnitude occurs within several degrees of sample tilting, that is two orders of magnitude larger. Moreover, the thickness of the HMM is $176 \mathrm{~nm}$ only as opposed to those of the dielectric materials: $50 \mu \mathrm{m}$ for the polymer film and $1 \mathrm{~mm}$ for the quartz plate.

In conclusion, we experimentally demonstrated for the first time the photonic spin Hall effect in a hyperbolic metamaterial at visible wavelengths. The tranverse beam shift in the transmission configuration is very sensitive to the incident angle: we observed that a few milliradian difference changes the beam shift by two orders of magnitude, for example, from a few hundreds of microns down to several microns. This extreme angular tunability is realized in a two hundreds of nanometers thick HMM. Such sensitivity can lead to thin and compact spin Hall devices that manipulate light at nanoscale by means of spin, incident angle, and wavelength, such as switches, filters, sensors.

Funding Information. Agencia Nacional de Promocion Cientifica y Tecnologica, PICT2015-0710 Startup, UBACyT PDE 2016, UBACyT PDE 2017, Argentina; Villum Fonden DarkSILD project (11116); Direktør Ib Henriksens Fond, Denmark.

Acknowledgments. The authors are grateful to Konstantin Bliokh, Mark Dennis, and Joerg Goette for fruitful discussions.

\section{REFERENCES}

1. Y. Liu, Y. Ke, H. Luo, and S. Wen, Nanophotonics 6, 51 (2017).

2. X. Ling, X. Zhou, K. Huang, Y. Liu, C.-W. Qiu, H. Luo, and S. Wen, Rep. Prog. Phys. 80, 066401 (2017).

3. N. B. Baranov, A. Y. Savchenko, and B. Y. Zel'dovich, JETP Lett. 59, 232 (1994).

4. Z. Bomzon, G. Biener, V. Kleiner, and E. Hasman, Opt. Lett. 27, 1141 (2002).

5. K. Y. Bliokh, Phys. Rev. Lett. 97, 1 (2006).

6. K. Y. Bliokh, A. Niv, V. Kleiner, and E. Hasman, Nat. Photonics 2, 748 (2008).

7. A. Aiello and J. P. Woerdman, Opt. Lett. 33, 1437 (2008).

8. K. Y. Bliokh and A. Aiello, J. Opt. (United Kingdom) 15 (2013).

9. K. Y. Bliokh, F. J. Rodríguez-Fortuño, F. Nori, and A. V. Zayats, Nat. Photonics 9, 796 (2015).

10. F. Cardano and L. Marrucci, Nat. Photonics 9, 776 (2015).

11. A. Aiello, G. Puentes, D. Voigt, and J.P. Woerdman, Phys. Rev. A 75, 062118 (2007).

12. X. Zhou, X. Ling, H. Luo, and S. Wen, Appl. Phys. Lett. 101, 251602 (2012).

13. X. Zhou, L. Sheng, and X. Ling, Sci. Reports 8, 1221 (2018).

14. Y. Qin, Y. Li, X. Feng, Z. Liu, H. He, Y.-F. Xiao, and Q. Gong, Opt. Express 18, 16832 (2010). X. Zhou and X. Ling, Opt. Express 24, 3025 (2016).
15. Y. Qin, Y. Li, H. He, and Q. Gong, Opt. Lett. 34, 2551 (2009).

16. X. Zhou, Z. Xiao, H. Luo, and S. Wen, Phys. Rev. A - At. Mol. Opt. Phys. 85, 1 (2012).

17. X. Zhou and X. Ling, Opt. Express 24, 3025 (2016).

18. B. Wang, Y. Li, M. M. Pan, J. L. Ren, Y. F. Xiao, H. Yang, and Q. Gong, Phys. Rev. A - At. Mol. Opt. Phys. 88, 5 (2013).

19. X. Zhou, J. Zhang, X. Ling, S. Chen, H. Luo, and S. Wen, Phys. Rev. A 88, 053840 (2013).

20. K. Y. Bliokh, C. T. Samlan, C. Prajapati, G. Puentes, N. K. Viswanathan, and F. Nori, Optica 3, 1039 (2016).

21. O. Takayama and G. Puentes, Opt. Lett. 43, 1343 (2018).

22. X. Zhou, X. Ling, Z. Zhang, H. Luo, and S. Wen, Sci. Reports 4, 1 (2014).

23. L. Marrucci, C. Manzo, and D. Paparo, Phys. Rev. Lett. 96, 1 (2006).

24. D. Haefner, S. Sukhov, and A. Dogariu, Phys. Rev. Lett. 102, 1 (2009).

25. A. J. Hoffman, L. Alekseyev, E. E. Narimanov, C. Gmachl, and D. L. Sivco, Nat. Mater. 6, 946 (2007).

26. H. N. S. Krishnamoorthy, Z. Jacob, E. Narimanov, I. Kretzschmar, and V. M. Menon, Sci. (New York, N.Y.) 336, 205 (2012). V. P. Drachev, Laser Photonics Rev. 7, 265 (2013).

27. K. V. Sreekanth, Y. Alapan, M. ElKabbash, E. Ilker, M. Hinczewski, U. A. Gurkan, A. De Luca, and G. Strangi, Nat. Mater. 15, 621 (2016).

28. O. Takayama, E. Shkondin, A. Bogdanov, M. E. Aryaee Pahah, K. Golenitskii, P. A. Dmitriev, T. Repän, R. Malreanu, P. Belov, F. Jensen, and A. V. Lavrinenko, ACS Photonics 4, 2899 (2017).

29. E. Shkondin, T. Repän, M. E. Aryaee Panah, A. V. Lavrinenko, and O. Takayama, ACS Appl. Nano Mater. 1, 1212 (2018).

30. O. Takayama, P. Dmitriev, E. Shkondin, O. Yermakov, M. E. A. Panah, K. Golenitskii, F. Jensen, A. Bogdanov, and A. V. Lavrinenko, Semiconductors. 52, 442 (2018).

31. M. A. Noginov, Y. A. Barnakov, G. Zhu, T. Tumkur, H. Li, and E. E. Narimanov, Appl. Phys. Lett. 94, 1 (2009).

32. V. Kabashin, P. Evans, S. Pastkovsky, W. Hendren, G. Wurtz, R. Atkinson, R. Pollard, V. Podolskiy, and V. Zayats, Nat. materials 8, 867 (2009).

33. Y. Guo, W. Newman, C. L. Cortes, and Z. Jacob, Adv. OptoElectronics 2012, 1 (2012).

34. A. Poddubny, I. Iorsh, P. Belov, and Y. Kivshar, Nat. Photonics 7, 948 (2013).

35. D. Lu and Z. Liu, Nat. Commun. 3, 1205 (2012).

36. T. Tang, C. Li, and L. Luo, Sci. Reports 6, 30762 (2016).

37. T. Tang, Y. Zhang, J. Li, and L. Luo, IEEE Photonics J. 9, 4600910 (2017).

38. P. V. Kapitanova, P. Ginzburg, F. J. Rodríguez-Fortuño, D. S. Filonov, P. M. Voroshilov, P. Belov, A. N. Poddubny, Y. S. Kivshar, G. Wurtz, and A. V. Zayats, Nat. communications 5, 3226 (2014).

39. J. Sukham, O. Takayama, A. Lavrinenko, and R. Malureanu, ACS Appl. Mater. Interfaces 9, 25049 (2017).

40. L. Ferrari, C. Wu, D. Lepage, X. Zhang, and Z. Liu, Prog. Quantum Electron. 40, 1 (2015).

41. A. Lavrinenko and V. Zhilko, Microw. Opt. Technol. Lett. 15, 54 (1997).

42. L. Brigo, E. Gazzola, M. Cittadini, P. Zilio, and G. Zacco, Nanotechnology. 24, 155502 (2013).

43. G. Puentes, N. Hermosa, and J. Torres, Phys. Rev. Lett. 109, 040401 (2012). 